\title{
LARGE-SCALE UNSTEADINESS IN A TWO-DIMENSIONAL DIFFUSER: NUMERICAL STUDY TOWARD ACTIVE SEPARATION CONTROL
}

\author{
Takao Suzuki and Tim Colonius ${ }^{\dagger}$ \\ Division of Engineering and Applied Science \\ California Institute of Technology, Pasadena, California 91125
}

\begin{abstract}
$\underline{\text { ABSTRACT }}$
We develop a reduced order model for large-scale unsteadiness (vortex shedding) in a two-dimensional diffuser and study the mechanisms of active flow separation control. This model can estimate the vortex shedding frequency for inviscid flows by accounting for the accumulated vorticity flux in the diffuser. The model can also predict the stagnation pressure loss, which consists of two parts: A steady part corresponds to static pressure loss on the detached area, and an unsteady part is associated with vortex shedding. To validate this model, we perform direct numerical simulation (DNS) of compressible, laminar diffuser flows. The comparison between the model and DNS shows good agreement at various Mach numbers and area ratios of the diffuser in terms of vortex shedding time scale and stagnation pressure loss. To investigate the effects of periodic mass injection near the separation point, we also perform DNS over a wide range of the forcing frequency. The DNS results show that periodic mass injection can pinch off vortices with a smaller size; accordingly, their convective velocity is increased, absorption of circulation from the wall is enhanced, and the extent of the separated region is reduced. As a result, the stagnation pressure recovery, particularly the unsteady part, is substantially improved as predicted by the model.
\end{abstract}

\section{INTRODUCTION}

To achieve high performance in aircraft propulsion systems, it is crucial to reduce stagnation pressure loss across an inlet diffuser. In many situations the length of the diffuser is restricted, e.g. lowobservability constraints and rapid area changes are required (c.f. Hamstra, et al. 2000; MacMartin, et al. 2001). As a result, large adverse pressure

Copyright (C)2003 by Takao Suzuki and Tim Colonius. Published by the American Institute of Aeronautics and Astronautics, Inc., with permission. gradients between the throat and compressor face cause boundary layer separation, i.e. stall. Previous experimental studies (e.g. Reneau, Johnston, \& Kline 1967) have been used to categorize stall regimes for a planar diffuser geometry. Particularly, in the so-called "transitory stall regime," the boundary layer separation is continuously built up and semi-periodically washed out; thus, the flow pattern looks similar to vortex shedding from a bluff body. Such a pattern has been visualized in experiments (Salmon, Bogar, \& Sajben 1983). This phenomenon leads to substantial reduction in stagnation pressure.

In past, a number of studies have demonstrated that the pressure recovery can be improved if large scale vortical disturbances are disrupted. In fact, some experimental studies successfully reduced the stagnation pressure loss, for example, by using splitter vanes (Rao 1971), steady mass injection (Nicoll \& Ramaprian 1970; Back \& Cuffel 1982; Nishi, Yoshida, \& Morimitsu 1998), a star tail-pipe (Welsh 1976), etc. In these studies, however, the optimal design parameters, such as the mass flow rate or the rotation rate of the actuator, were only empirically studied. In this study, we consider periodic mass injection in order to simulate a synthetic jet (c.f. Glezer \& Amitay 2002). This technique was initially introduced for airfoil separation control (e.g. Seifert, Darabi, \& Wygnanski 1996) and has been recently applied to internal flow separation control (e.g. Narayanan \& Banaszuk 2003, and others). These studies have found that periodic separation enhances pressure recovery more than previous active flow control techniques. Thus, the objective of this research is to investigate the large-scale flow unsteadiness intrinsic to transitory stall in a twodimensional diffuser and to study the mechanisms of the periodic mass injection technique to control flow separation.

We develop a compressible inviscid model to scale the vortex shedding time period by accounting for the accumulated vorticity flux in a diffuser. We also estimate the stagnation pressure loss based on an 


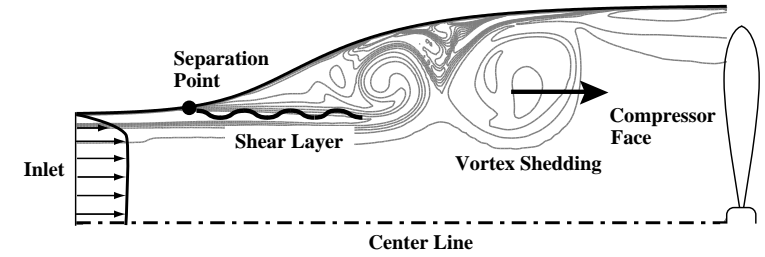

Figure 1: Schematic of vortex shedding in a twodimensional diffuser.

ideal fluid. This model consists of steady and unsteady parts, the former accounts for static pressure loss on the separated region and the latter for vortex shedding. This model provides implications for reducing stagnation pressure loss. Based on this model, we study the frequency dependence of periodic mass injection near the separation point.

To verify the theoretical analyses, we perform direct numerical simulations (DNS) of twodimensional laminar diffuser flows at various inflow Mach numbers $\left(M_{1}=0.2 \sim 0.8\right)$ and the diffuser area ratios $\left(h_{2} / h_{1}=1.4 \sim 2.6\right)$ : We compare the shedding time period calculated from DNS and the time scale given by the theory. We also compare the stagnation pressure loss between DNS and the incompressible model. To investigate the effects of periodic mass injection, we locally force the boundary layer at several different frequencies and analyze each component of the stagnation pressure loss based on DNS in detail.

The outline of the paper is as follows: In the next section, the vortex shedding model is introduced, and the stagnation pressure loss is estimated in the incompressible limit. Next, the procedures of the numerical simulations are presented. Subsequently, the DNS results are compared with the theories.

\section{THEORETICAL APPROACH}

\section{MECHANISM OF VORTEX SHEDDING}

To estimate the vortex shedding frequency, we consider a simple two-dimensional symmetric diffuser geometry (see figure 2 for the coordinate system) and assume an inviscid fluid. We take a control volume as shown in figure 2 and calculate the accumulated vorticity flux per unit time. In compressible flows it is appropriate to calculate the flux of $\omega / \rho$ (c.f. section 1.5 of Saffman 1992). Recall that Kelvin's circulation theorem ensures that circulation is neither generated nor dissipated in an inviscid (and barotropic) fluid. Of course, some vorticity flux is generated or absorbed through the diffuser wall due to the non-slip boundary condition, but this will be taken into account later.

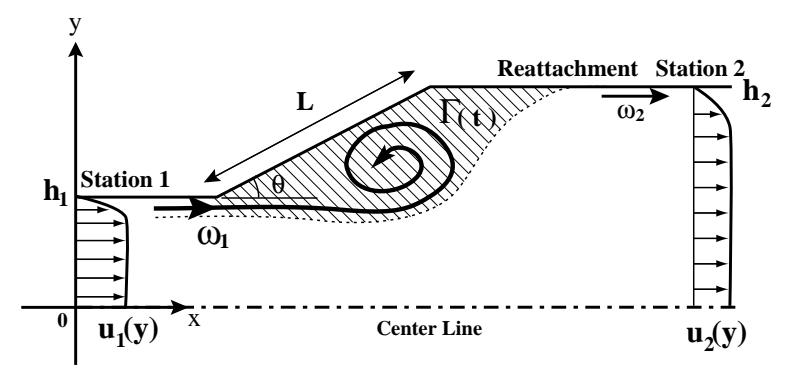

Figure 2: Simple two-dimensional diffuser model.

We assume that the inflow velocity and temperature profiles are transversely sheared, denoted by $u_{1}(y)$ and $T_{1}(y)$, respectively. The inflow pressure is assumed constant (denoted by $p_{1}$ ); consequently, the density is given from the ideal gas law as $\rho_{1}(y)$. Furthermore, we assume that in the diffuser the separated boundary layer reattaches before it reaches the out-flow boundary (station 2 in figure 2). The exit conditions are similarly assumed, and they are expressed using the subscript 2 ( $\operatorname{such}$ as $u_{2}(y)$ as the exit velocity profile). Consequently, the net circulation (per density) accumulated in the control volume per unit time can be calculated as

$$
\begin{aligned}
\frac{1}{\bar{\rho}} \frac{d \Gamma}{d t} & \equiv \int_{0}^{h_{1}} \frac{\omega_{1}(y)}{\rho_{1}(y)} u_{1}(y) d y-\int_{0}^{h_{2}} \frac{\omega_{2}(y)}{\rho_{2}(y)} u_{2}(y) d y \\
& =\frac{R}{p_{1}} \int_{0}^{u_{1}} T_{1}\left(u_{1}\right) u_{1} d u_{1}-\frac{R}{p_{2}} \int_{0}^{u_{2}} T_{2}\left(u_{2}\right) u_{2} d u_{2},(1)
\end{aligned}
$$

where $\bar{\rho}$ on the left hand side denotes a characteristic density scale, and $R$ the universal gas constant.

To evaluate the temperature profile, we use the Crocco-Busemann relation (c.f. section XV of Schlichting 1960). For an iso-thermal wall, the temperature profile can be calculated as

$$
\begin{array}{r}
\frac{T(u)}{T_{\infty}}=\frac{T_{\text {wall }}}{T_{\infty}}+\left(1-\frac{T_{\text {wall }}}{T_{\infty}}+\frac{u_{\infty}^{2}}{2 c_{p} T_{\infty}}\right) \frac{u}{u_{\infty}} \\
-\frac{u_{\infty}^{2}}{2 c_{p} T_{\infty}}\left(\frac{u}{u_{\infty}}\right)^{2},
\end{array}
$$

where $c_{p}$ represents the specific heat at constant pressure $(\equiv \gamma R /(\gamma-1), \gamma$ being the specific heat ratio). In addition, the subscript $\infty$ denotes the free-stream quantity (i.e. above the boundary layer), which is assumed identical to the center-line quantity, and $T_{\text {wall }}$ is the wall temperature, which is assumed constant everywhere. Recall that (2) is derived assuming that the temperature is a function of velocity only and the Prandtl number is unity. Now, substituting (2) into (1) yields 


$$
\begin{aligned}
\frac{1}{\bar{\rho}} \frac{d \Gamma}{d t} & =\frac{u_{1}^{2}}{2 \rho_{1}}\left[\frac{2}{3}+\frac{1}{3} \frac{T_{\text {wall }}}{T_{1}}+\frac{(\gamma-1) M_{1}^{2}}{12}\right] \\
& -\frac{u_{2}^{2}}{2 \rho_{2}}\left[\frac{2}{3}+\frac{1}{3} \frac{T_{\text {wall }}}{T_{2}}+\frac{(\gamma-1) M_{2}^{2}}{12}\right] .
\end{aligned}
$$

Here, $M_{j} \equiv u_{j} / \sqrt{\gamma R T_{j}}(j=1$ or 2$)$ is the Mach number, and the quantities without $(y)$ denote the center-line quantities (such as, $u_{1} \equiv u_{1}(0)$ ). It is important to notice that the net vorticity flux (per unit density) is independent of the velocity profile.

During one period of vortex shedding (referred to as $\left.T_{\text {shed }}\right)$, we assume that the accumulated circulation forms a single vortex in the hatched region in figure 2. In a viscous flow some vorticity flux is absorbed from the wall, and this rate is expressed by $\lambda$ (which will be formulated in (22) later). Now, we assume that the diameter of the vortex can be scaled as $\left(h_{2}-h_{1}\right)$ and its averaged density $\bar{\rho}$ in (3) as $\rho_{1}$ (in fact, it is even lower due to compressibility). Using Stokes' theorem, the averaged velocity at the outer radius of this vortex can be estimated as

$$
\bar{u} \approx \frac{(1-\lambda) \frac{\rho_{1}}{\bar{\rho}} \frac{d \Gamma}{d t} \cdot T_{\text {shed }}}{\pi\left(h_{2}-h_{1}\right)} .
$$

When this velocity exceeds some velocity scaled by the free-stream velocity (denoted by $\alpha u_{1}$ ), we hypothesize that the vortex is pinched off. Note that as the area ratio becomes greater, the vortex formation criterion suggested by Gharib, Rambod, \& Shariff (1998) may be applied. Now, the time period of vortex shedding can be then estimated as

$$
T_{\text {shed }} \approx \alpha \tau_{\text {shed }} \equiv \frac{\alpha}{1-\lambda} \frac{2 \pi h_{1}\left(\frac{h_{2}}{h_{1}}-1\right)}{u_{1} F}
$$

where

$$
\begin{aligned}
F\left(M_{1}, \frac{h_{2}}{h_{1}}, \frac{u_{2}}{u_{1}}, \frac{T_{2}}{T_{1}}, \frac{T_{\text {wall }}}{T_{1}}\right) \\
\equiv\left[\frac{1}{2}+\frac{1}{3} \frac{T_{\text {wall }}}{T_{1}}+\frac{1}{6}\left(1+\frac{\gamma-1}{2} M_{1}^{2}\right)\right]-\left(\frac{h_{2}}{h_{1}}\right)\left(\frac{u_{2}}{u_{1}}\right)^{3} \\
\times\left[\frac{1}{2}+\frac{1}{3} \frac{T_{\text {wall }}}{T_{1}} \frac{T_{1}}{T_{2}}+\frac{1}{6}\left(1+\frac{\gamma-1}{2} M_{1}^{2}\right) \frac{T_{1}}{T_{2}}\right] .
\end{aligned}
$$

Here, it is assumed that the effects of the boundary layer are sufficiently small so that the center-line quantities follow the continuity and the energy conservation given from a quasi-one-dimensional analysis. It is also noted that the velocity and temperature ratios are determined from the area ratio assuming the ideal expansion and the quasi-onedimensionality. This is equivalent to treating a subscript as a cross section average.

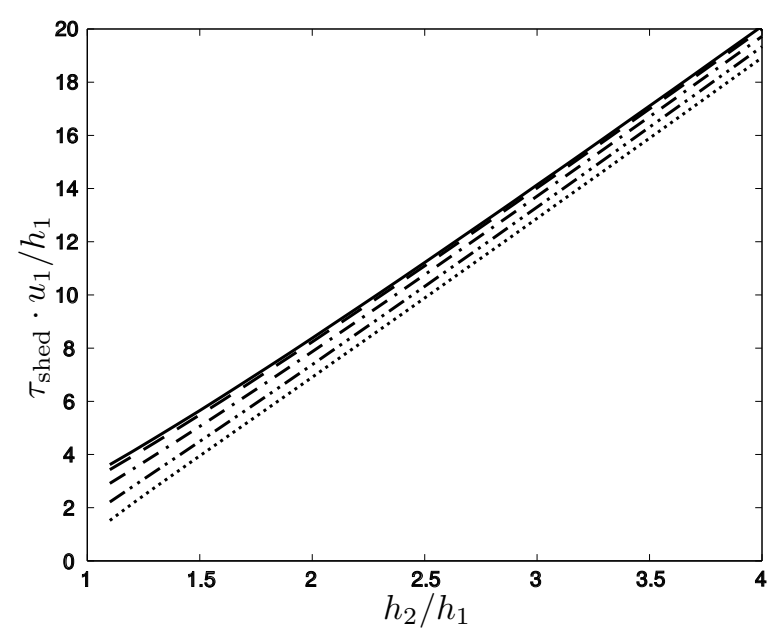

Figure 3: Compressible effect on the vortex shedding time period. The vortex shedding time period versus the area ratio at various inflow Mach numbers are shown: - Incompressible limit;,$--- M_{1}=$ $0.2 ;-\cdots-\cdot-, M_{1}=0.4 ;-\cdots-\cdots-, M_{1}=0.6 ; \cdots$, $M_{1}=0.8$. The absorption of circulation from the wall is neglected $(\lambda=0)$.

Notice that in incompressible flows, (5) yields the following simple expression:

$$
T_{\text {shed }} \approx \alpha \tau_{\text {shed }}^{(M \rightarrow 0)} \equiv \frac{\alpha}{1-\lambda} \frac{2 \pi h_{1}\left(\frac{h_{2}}{h_{1}}\right)^{2}}{u_{1}\left(1+\frac{h_{2}}{h_{1}}\right)}
$$

where we assume that $\rho_{1}=\rho_{2}, T_{1}=T_{2}=T_{\text {wall }}$, and $M_{1}=0$. Figure 3 plots the shedding time period as a function of the area ratio at various inflow Mach numbers. Here, $p_{2} / p_{1}$ is calculated based on the ideal expansion, and $T_{\text {wall }}=T_{1}$ is assumed. It shows that the shedding period becomes longer as the area ratio increases.

\section{STAGNATION PRESSURE LOSS STEADY PART}

In this section we evaluate stagnation pressure loss due to flow separation and large scale flow unsteadiness in an incompressible limit. We start with Crocco's equation:

$$
\frac{\partial u_{i}}{\partial t}+\frac{\partial}{\partial x_{i}}\left(h+\frac{u_{k}^{2}}{2}\right)-T \frac{\partial s}{\partial x_{i}}=\epsilon_{i j k} u_{j} \omega_{k}+\frac{1}{\rho} \frac{\partial \tau_{i j}}{\partial x_{j}}
$$

where $h$ is enthalpy $\left(\equiv c_{p} T\right), s$ is entropy, and $\tau_{i j}$ is the viscous stress tensor. Defining stagnation temperature to be $T_{t} \equiv\left(h+\frac{u_{j}^{2}}{2}\right) / c_{p}$, and accordingly, stagnation pressure and density to be 
$p_{t} \equiv\left(T_{t} / T\right)^{\frac{\gamma}{\gamma-1}} p$ and $\rho_{t} \equiv\left(T_{t} / T\right)^{\frac{1}{\gamma-1}} \rho$, respectively, (8) can be rewritten as

$$
\frac{\partial p_{t}}{\partial x_{i}}=\rho_{t} \epsilon_{i j k} u_{j} \omega_{k}-\rho_{t} \frac{\partial u_{i}}{\partial t}+\left(T-T_{t}\right) \rho_{t} \frac{\partial s}{\partial x_{i}}+\frac{\rho_{t}}{\rho} \frac{\partial \tau_{i j}}{\partial x_{j}}
$$

Note that entropy is the same between static and stagnation quantities by definition. Now, we evaluate the order of each term in (9). Here, we assume that the compressibility is weak and expand both static and stagnation thermodynamic quantities for small $M^{2}$. For example, $p \approx p_{\infty}+M^{2} \tilde{p}_{M^{2}}+$ $M^{4} \tilde{p}_{M^{4}}+\cdots$, etc. In addition, we assume that the time scale of the fluid motion is proportional to $1 / M$, namely $\left(\frac{\partial}{\partial t}\right) /\left(\frac{\partial}{\partial x}\right) \sim O(M)$. The first two terms on the right hand side of (9) then yield $O\left(M^{2}\right)$. The third term can be estimated as $O\left(M^{4} / R e\right)$ referring to the entropy change along the material line (if the flow is not initially homoentropic, its order is $\left.O\left(M^{4}\right)\right)$. Likewise, the last term can be evaluated as $O\left(M^{2} / R e\right)$. Thus, assuming $R e \gg 1$ and neglecting $O\left(M^{4}\right)$ terms, (9) can be approximated as

$$
\frac{\partial p_{t}}{\partial x_{i}} \approx \rho_{t} \epsilon_{i j k} u_{j} \omega_{k}-\rho_{t} \frac{\partial u_{i}}{\partial t}
$$

Next, we integrate (10) for $i=1$ (the $x$ direction) inside the control volume defined in figure 2. Using Green's theorem, the left hand side yields the surface integrals. Integrating over the cross section, the second term on the right hand side vanishes for a symmetric vortex, as discussed later. Alternatively, if we take a time average and expand this term as $\overline{\left(\rho_{\infty}-\rho\right) \frac{\partial u}{\partial t}}-\overline{\rho_{\infty} \frac{\partial u}{\partial t}}$, it is evaluated as $O\left(M^{4}\right)$. Consequently, the stagnation pressure loss averaged in the vertical direction can be approximated as

$$
p_{t 1}-p_{t 2} \approx\left(p_{t 1}-p_{w}\right)\left(1-\frac{h_{1}}{h_{2}}\right)-\frac{\iint \rho_{t} v \omega d x d y}{h_{2}},
$$

where the averaged pressure projected on the wall is defined as $p_{w} \equiv \int_{h_{1}}^{h_{2}} p d y /\left(h_{2}-h_{1}\right)$. In a fully separated diffuser, $p_{w} \approx p_{1}$. As mentioned in the previous section, quantities with a subscript denote the cross section average. Figure 4 shows the error of (11) assuming $p_{w}=p_{1}$. Slight under-estimate of pressure on the wall $(\sim 5 \%)$ causes over-estimate of the stagnation pressure loss. Nonetheless, the time variation of the error in (11) is relatively small (the averaged error during $t u_{1} / h_{1} \in[24,120]$ in figure 4 is approximately $-2.1 \%$ ). Thus, our strategy to suppress the stagnation pressure loss is to increase $p_{w}$ and $\iint \rho_{t} v \omega d x d y$.

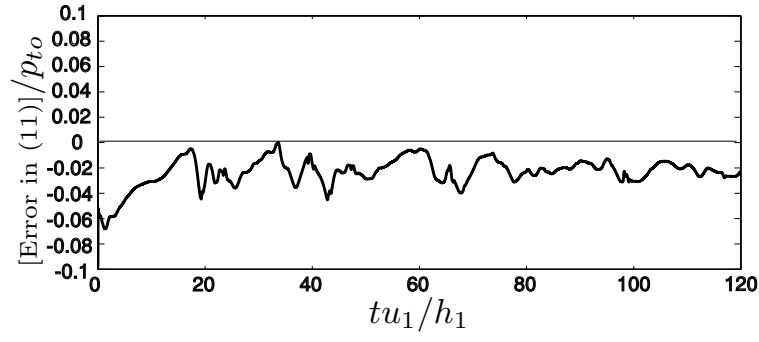

Figure 4: Error in the estimate of stagnation pressure loss. Referring to (11), (L.H.S. - R.H.S.) $/ p_{t o}$ is plotted, where $p_{t o} \equiv\left(1+\frac{\gamma-1}{2} M_{1}^{2}\right)^{\frac{\gamma}{\gamma-1}} p_{1}$. Correction of the projected area noted in table 1 is also taken into account.

Now, the steady part of $\iint \rho_{t} v \omega d x d y$ can be readily evaluated in incompressible flows. Imposing the non-slip boundary conditions on the wall, this term can be expanded as follows:

$$
\begin{aligned}
& \iint \rho_{t} v \omega d x d y \approx \iint \rho_{\infty} v\left(\frac{\partial v}{\partial x}-\frac{\partial u}{\partial y}\right) d x d y \\
= & -\rho_{\infty} \iint \frac{\partial\left(u^{2} / 2\right)}{\partial x} d x d y=\rho_{\infty} \frac{u_{1}^{2} h_{1}-u_{2}^{2} h_{2}}{2}
\end{aligned}
$$

Here, continuity is used, and $v_{1}=v_{2}=0$ is assumed for the third equality. This relation is nothing but a momentum balance of the control volume. Thus, the steady part of the time averaged stagnation pressure loss can be expressed as

$\left(\overline{p_{t 1}-p_{t 2}}\right)_{\text {steady }}^{M \rightarrow 0} \approx \frac{\left(p_{1}-\overline{p_{w}}\right)\left(\frac{h_{2}}{h_{1}}-1\right)}{\frac{h_{2}}{h_{1}}}+\frac{\rho u_{1}^{2}}{2} \frac{\left(\frac{h_{2}}{h_{1}}-1\right)^{2}}{\left(\frac{h_{2}}{h_{1}}\right)^{2}}$

\section{UNSTEADY PART}

The unsteady part of the $\iint \rho_{t} v \omega d x d y$ term is considered to be the contribution from vortex shedding. To analyze this effect, we consider the Oseen vortex (Oseen 1912) as a model problem, which is a solution to the incompressible Navier-Stokes equations. Again, we consider the leading terms of the incompressible limit. The radial velocity of the Oseen vortex is given by

$$
\begin{aligned}
u_{r}(t, x, y) & =\frac{\Gamma}{2 \pi \sqrt{\left(x-x_{o}\right)^{2}+\left(y-y_{o}\right)^{2}}} \\
& \times\left(1-\exp \left[-\frac{\left(x-x_{o}\right)^{2}+\left(y-y_{o}\right)^{2}}{4 \nu t}\right]\right),
\end{aligned}
$$


where $\left(x_{o}, y_{o}\right)$ denotes the center of the vortex, $\Gamma$ is the total circulation, and $\nu$ is the kinematic viscosity. Here, we assume that the time scale of dissipation is much slower than that of vortex convection; accordingly, we define the radius of the vortex to be $R \equiv \sqrt{4 \nu t}$ and assume it to be constant. Using this expression and assuming that the effect of blockage due to the wall is weak, we obtain the following quantity after some algebra:

$$
\begin{aligned}
& \int_{-\infty}^{\infty} \rho_{t} v \omega d y \\
& =\frac{\rho_{t} \Gamma^{2}}{2 \pi R^{2}}\left(\operatorname{erf}\left[\frac{\sqrt{2}\left(x-x_{o}\right)}{R}\right]-\operatorname{erf}\left[\frac{x-x_{o}}{R}\right]\right)
\end{aligned}
$$

where $\operatorname{erf}(z) \equiv \frac{2}{\sqrt{\pi}} \int_{0}^{z} e^{-t^{2}} d t$. Notice that (15) is positive downstream of the vortex center $\left(x-x_{o}>0\right)$ and negative upstream of it $\left(x-x_{o}<0\right)$. Therefore, when the Oseen vortex leaves the control volume, a stagnation pressure drop appears at the exit cross section. According to the discussion in the previous section, we estimate the parameters as $\Gamma=\alpha 2 \pi R u_{1}$ and $R \approx\left(h_{2}-h_{1}\right) / 2$ and express the convective velocity of the vortex to be $u_{c}$. Thus, the stagnation pressure loss averaged over the exit cross section is given as a function of time,

$$
\begin{aligned}
& \left(\widetilde{p_{t 2}}\right)_{\text {shed }}(t)=\frac{1}{h_{2}} \int_{u_{c} t}^{\infty} \int_{-\infty}^{\infty} \rho_{t} v \omega d y d x \\
& \approx \alpha^{2} \pi \rho_{\infty} u_{1}^{2} \frac{h_{2}-h_{1}}{h_{2}} \int_{\infty}^{\frac{u_{c} t}{R}}(\operatorname{erf}[\sqrt{2} \chi]-\operatorname{erf}[\chi]) d \chi
\end{aligned}
$$

When the vortex is convected along $y=h_{2} / 2$, the convective velocity can be approximated as $u_{c}=u_{2}$. In reality, the vortex is pinched off near the wall and convected above $y=h_{2} / 2$ when $h_{2} / h_{1}<2$, and vice versa. Therefore, the convective velocity is reduced due to the induced velocity. To be precise, the Oseen vortex is no longer the solution because the center of the vortex is convected slower than the mean flow in addition to the distortion due to the wall.

To estimate the induced velocity due to blockage, we assume an ideal fluid and consider a conformal mapping represented by $\zeta=\exp \left(\frac{\pi}{2 h_{2}} z\right)$. This function maps an infinitely long channel onto the first quadrant; hence, two pairs of counter-rotating point vortices satisfy the non-penetration boundary condition in the $\zeta$ plane. According to the study by Pierrehumbert (1980) based on a pair of counter-rotating vortex 'patches,' convective velocities of distributed vortices can be well approximated by point vortices even if the distance between them is relatively small.
Based on point vortices, the rate of induced velocity can be calculated as (c.f. section 7.2 of Saffman 1992)

$$
\kappa\left(\frac{h_{2}}{h_{1}}, \frac{h_{3}}{h_{1}}\right) \equiv-\frac{u_{\text {ind }}}{\alpha u_{2}} \approx-\frac{\pi}{4}\left(\frac{h_{2}}{h_{1}}-1\right) \frac{1}{\tan \left(\pi \frac{h_{3}}{h_{1}} \frac{h_{1}}{h_{2}}\right)},
$$

where $h_{3}$ is the height of the vortex from the bottom, and the convective velocity then becomes $(1-\alpha \kappa) u_{2}$. For example, when $h_{2} / h_{1}=2$ and $h_{3} / h_{1}=\left(\left(h_{1}+\right.\right.$ $\left.\left.h_{2}\right) /\left(2 h_{1}\right)+h_{2} / h_{1}\right) / 2=5 / 4, \kappa$ becomes as high as 0.33 (for reference, if we simply calculate the infinite superposition of the Oseen vortices, $\kappa$ yields 0.32 , which is sufficiently close to the point vortex solution). Thus, the effect of blockage on the convective velocity is not negligible. On the other hand, the error of $\iint \rho_{t} v \omega d x d y$ associated with blockage is less than $2 \%$.

Using (16) and knowing that the time period of vortex shedding is estimated by (7), the time averaged stagnation pressure loss associated with vortex shedding can be estimated as

$$
\begin{aligned}
\left(\widetilde{\widetilde{p_{t 2}}}\right)_{\text {shed }}^{M \rightarrow 0} & =\frac{\int_{-\infty}^{\infty} \widetilde{p_{t 2}}(t) d t}{T_{\text {shed }}^{(M \rightarrow 0)}} \\
& \approx-\frac{\alpha(1-\lambda)}{1-\alpha \kappa} \frac{\left(\frac{h_{2}}{h_{1}}+1\right)\left(\frac{h_{2}}{h_{1}}-1\right)^{2}}{8\left(\frac{h_{2}}{h_{1}}\right)^{2}} \frac{\rho u_{1}^{2}}{2} .
\end{aligned}
$$

This equation shows that the stagnation pressure loss due to vortex shedding is proportional to the dynamic pressure in the incompressible limit. This expression is also valid when we artificially tune $\alpha$; therefore, by breaking the circulation into smaller vortices, the stagnation pressure loss can be suppressed. Although we have derived (18) based on the Oseen vortex, this dependence is still valid when the vortex is radially symmetric. We define a coefficient relative to the Oseen vortex (i.e. in front of (18)) as $\beta$. In fact, $\beta$ is found to be reasonably close to unity in DNS (see table 3 shown later).

Accordingly, the total stagnation pressure loss is now expressed as

$$
\begin{aligned}
\left(\overline{p_{t 1}-p_{t 2}}\right)_{\mathrm{total}}^{M \rightarrow 0} \approx \frac{\left(p_{1}-\overline{p_{w}}\right)\left(\frac{h_{2}}{h_{1}}-1\right)}{\frac{h_{2}}{h_{1}}} \\
+\frac{\rho u_{1}^{2}}{2} \frac{\left(\frac{h_{2}}{h_{1}}-1\right)^{2}}{\left(\frac{h_{2}}{h_{1}}\right)^{2}}\left[1+\frac{\alpha \beta(1-\lambda)}{1-\alpha \kappa} \frac{\frac{h_{2}}{h_{1}}+1}{8}\right]
\end{aligned}
$$




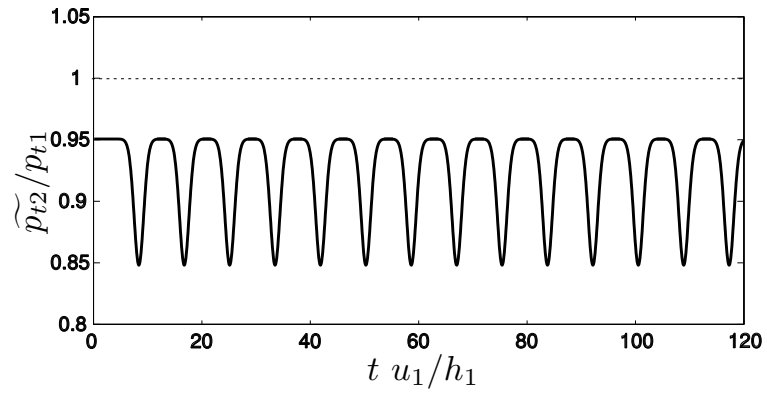

Figure 5: Stagnation pressure profile estimated based on the inviscid and incompressible limit. Parameters are chosen according to Case $\mathbf{A}\left(M_{1}=0.6\right.$ and $\left.h_{2} / h_{1}=2\right)$, and $\alpha=1, \beta=1, \lambda=0, p_{w}=p_{1}$, and $\kappa=0.33$ are assumed.

The first term and the first part of the second term correspond to the steady loss, and the second part of the second term to the unsteady loss. As discussed later, (19) indicates that the contribution from vortex shedding becomes comparable to the steady part as the induced velocity decelerates the convective velocity. Figure 5 shows an expected pattern of the stagnation pressure profile for a fully separated case. The troughs correspond to the unsteady loss repeated on the top of the steady part. The stagnation pressure drops when the center of the vortex is passing through the exit cross section (approximately $11 \%$ drop under the conditions corresponding to Case $\mathbf{A}$ defined in table 1). These theoretical predictions are examined using DNS.

\section{VORTICITY ABSORPTION FROM WALL}

Now, we consider viscous effects. In a free space, the viscous term simply diffuses the vorticity distribution but does not increase nor decrease net vorticity. Therefore, the dominant part of viscous effects should come from the vorticity flux on the wall. According to the previous discussion, the net accumulated circulation per unit time can be symbolically expressed as

$$
\begin{aligned}
\left(\frac{d \Gamma}{d t}\right)_{\text {total }} \approx \int_{\text {inflow }} & -\int_{\text {exit }}(\text { vorticity flux }) d y \\
+ & \int_{\text {wall }}(\text { vorticity flux }) d s
\end{aligned}
$$

To be precise, in compressible flows we must calculate flux of $\omega / \rho$ instead of "vorticity flux" as mentioned before. Thus, we assume that $\left(\frac{d \Gamma}{d t}\right)$ can be approximated by $\left(\frac{\rho_{1}}{\bar{\rho}} \frac{d \Gamma}{d t}\right)$ as defined in (1). For the second term, vorticity flux coming from the wall can be expressed as (e.g. Koumoutsakos, Leonard, \& Ṕepin 1994) $\int_{\text {wall }}($ vorticity flux $) d s=\int_{\text {wall }} \nu \frac{\partial \omega}{\partial n} d s=-\int_{\text {wall }} \frac{1}{\rho} \frac{\partial p}{\partial s} d s$

where the positive sign is taken to be production. This expression is valid even in compressible flows when the dynamic viscosity $\mu(=\rho \nu)$ is constant everywhere. This term produces additional circulation in favorable pressure gradients, while it absorbs circulation in adverse ones.

In incompressible flows, (21) simply yields $\left(p_{1}-p_{2}\right)_{\text {wall }} / \rho$. Assuming $\left(p_{1}\right)_{\text {wall }}=p_{1}$, the rate of absorption introduced in (4) can be expressed as

$$
\lambda \equiv-\frac{p_{1}-\left(\overline{p_{2}}\right)_{\mathrm{wall}}}{\rho \frac{d \Gamma}{d t}}=\frac{\left(\overline{p_{2}}\right)_{\mathrm{wall}}-p_{1}}{\frac{\rho u_{1}^{2}}{2}} \frac{\left(\frac{h_{2}}{h_{1}}\right)^{2}}{\left(\frac{h_{2}}{h_{1}}\right)^{2}-1}
$$

From the previous discussion and Bernoulli's equation, the rate of accumulated circulation per unit time in the control volume is $\frac{d \Gamma}{d t}=\frac{u_{1}^{2}-u_{2}^{2}}{2}=\frac{p_{2}-p_{1}}{\rho}$; therefore, if this amount is perfectly absorbed from the wall (i.e. $\lambda=1$ ) and the pressure on the wall coincides with the free-stream pressure, no vortex shedding occurs. However, when the flow is fully separated, vorticity is absorbed mainly near the reattachment point and the projection of recovered static pressure in the $x$ direction is reduced.

\section{NUMERICAL PROCEDURES}

DIRECT NUMERICAL SIMULATION (DNS)

To numerically investigate diffuser flows, we performed direct numerical simulations (DNS) solving the full compressible Navier-Stokes equations in twodimensions. For time marching the fourth order Runge-Kutta scheme was used. For spatial derivatives the sixth order Padé scheme (Lele 1992) was used for the interior points. For the inflow and exit boundary points, lower order (third and fourth order) Padé schemes were used. The rest of the boundary treatments are described later in this section.

To generate the computational grid, we used a conformal mapping. The following function gives the two-dimensional diffuser shape used in this study:

$$
z=\frac{\frac{h_{2}}{h_{1}}+1}{2} \bar{\zeta}+\frac{1}{c} \frac{\frac{h_{2}}{h_{1}}-1}{2} \log (\cosh (c \bar{\zeta})),
$$

where $z=x+i y$ corresponds to the physical domain and $\bar{\zeta}=\bar{\xi}+i \bar{\eta}$ to an intermediate computational domain. Furthermore, this computational domain was 
AIAA Paper 2003-1138

\begin{tabular}{c|cccc} 
Case & $M_{1}$ & $h_{2} / h_{1}$ & \multicolumn{2}{c}{$\theta_{\max } \& c$} \\
\hline $\mathbf{A}$ & 0.6 & $2.0(1.843)$ & $18.43^{\circ}, 0.7576$ \\
$\mathbf{B}$ & 0.2 & $2.0(1.843)$ & $18.43^{\circ}, 0.7576$ \\
$\mathbf{C}$ & 0.4 & $2.0(1.843)$ & $18.43^{\circ}, 0.7576$ \\
$\mathbf{D}$ & 0.8 & $2.0(1.843)$ & $18.43^{\circ}, 0.7576$ \\
$\mathbf{E}$ & 0.6 & $1.4(1.346)$ & $10.78^{\circ}$, & 0.8440 \\
$\mathbf{F}$ & 0.6 & $1.6(1.514)$ & $14.03^{\circ}, 0.8110$ \\
$\mathbf{G}$ & 0.6 & $2.6(2.320)$ & $22.31^{\circ}$, & 0.6992 \\
$\mathbf{I} \sim \mathbf{O}$ & 0.6 & $2.0(1.843)$ & $18.43^{\circ}$, & 0.7576 \\
\hline \hline Case & $R e$ & $\delta_{b . l .} / h_{1}$ & $\left(N_{x}, N_{y}\right)$ & $\left(\Delta t a_{2} / h_{2}\right)$ \\
\hline $\mathbf{A}$ & 4000 & 0.10 & 601,151 & $1.0 \times 10^{-3}$ \\
$\mathbf{B}$ & 5000 & 0.10 & 601,151 & $1.0 \times 10^{-3}$ \\
$\mathbf{C}$ & 4000 & 0.10 & 601,151 & $1.0 \times 10^{-3}$ \\
$\mathbf{D}$ & 6000 & 0.10 & 801,201 & $0.8 \times 10^{-3}$ \\
$\mathbf{E}$ & 8000 & 0.07 & 721,181 & $1.0 \times 10^{-3}$ \\
$\mathbf{F}$ & 6670 & 0.08 & 601,151 & $1.0 \times 10^{-3}$ \\
$\mathbf{G}$ & 2900 & 0.13 & 721,181 & $0.727 \times 10^{-3}$ \\
$\mathbf{I} \sim \mathbf{O}$ & 4000 & 0.10 & 601,151 & $1.0 \times 10^{-3}$ \\
\hline
\end{tabular}

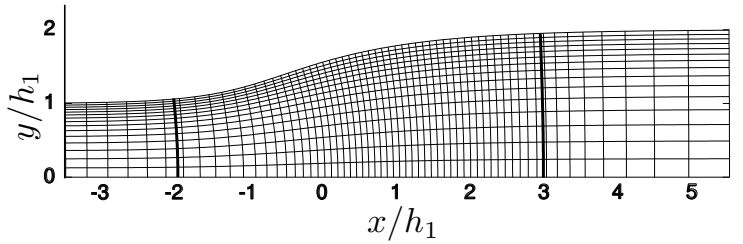

Figure 6: Typical computational grid. Every five grid point is shown for Case $\mathbf{A}$. Thicker lines denote the sections where the stagnation pressure profiles were recorded. $\Delta x_{\min } / h_{1}=8.29 \times 10^{-3}$, $\Delta x_{\max } / h_{1}=59.45 \times 10^{-3}, \Delta y_{\min } / h_{1}=5.57 \times 10^{-3}$, and $\Delta y_{\max } / h_{1}=25.15 \times 10^{-3}$.

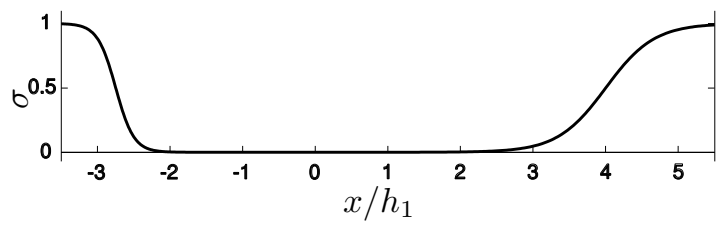

Figure 7: Strength of the sponge term.

Table 1: Flow conditions and diffuser geometries for DNS. The values in parentheses in the $h_{2} / h_{1}$ column denote the actual area ratio between the cross sections at which stagnation pressure profiles were recorded (see figure 6). The Reynolds number is defined as $R e \equiv u_{1} h_{1} / \nu_{1} . \delta_{b . l}$. denotes the inlet momentum thickness. $N_{x}$ and $N_{y}$ denote the numbers of grid points in the $x$ and $y$ directions, respectively, and $\Delta t$ is the time step.

linearly stretched to efficiently resolve the boundary layer near the wall as well as the region in which vortex stretching becomes intense. Consequently, spatial differentiation was performed in an equally spaced rectangular grid. A typical computational grid is shown in figure 6. Parameters for computation and the flow geometries are all tabulated in table 1.

According to the conformal mapping, initial velocity fields were calculated based on the potential flow solution, and the thermodynamic quantities were given from the compressible Bernoulli's equation. The initial velocity and thermodynamic quantity profiles near the wall were given by solving the Blasius boundary layer equation (c.f. Schlichting 1960) ignoring the curvature of the wall.

For the wall (the upper side of the computational domain), the non-slip boundary conditions were imposed, and the temperature distribution on the wall was calculated assuming the ideal expansion and set constant in time. The bottom line was set as the center-line assuming symmetry. At the inflow and the exit, non-reflecting boundary conditions (Poinsot \& Lele 1992) were imposed together

with a "sponge" buffer zone (Freund 1997). The coefficient $\sigma$ corresponding to the strength of the sponge is plotted in figure 7 .

\section{PERIODIC MASS INJECTION}

To study the effects of a synthetic jet, we artificially forced the right hand side of the Navier-Stokes equations and periodically injected a mass flow near the separation point in the $x$ direction:

$$
\begin{aligned}
& \frac{\partial \rho}{\partial t}+\frac{\partial\left(\rho u_{j}\right)}{\partial x_{j}}=F\left(x_{1}, x_{2}\right) \rho u_{s}(t) \\
& \frac{\partial\left(\rho u_{i}\right)}{\partial t}+\frac{\partial\left(\rho u_{i} u_{j}+p \delta_{i j}+\tau_{i j}\right)}{\partial x_{j}}=F\left(x_{1}, x_{2}\right) \rho u_{s}^{2}(t) \delta_{i 1} \\
& \frac{\partial\left[\rho\left(e+\frac{u_{k}{ }^{2}}{2}\right)\right]}{\partial t}+\frac{\partial\left[\left\{\rho\left(e+\frac{u_{k}{ }^{2}}{2}\right)+p\right\} u_{j}+\tau_{j k} u_{k}+q_{j}\right]}{\partial x_{j}} \\
& =F\left(x_{1}, x_{2}\right) \rho\left(e+\frac{u_{s}^{2}(t)}{2}\right) u_{s}(t),
\end{aligned}
$$

where

$$
\begin{aligned}
F(x, y) & =\frac{A_{o}}{2 \pi \sigma_{x} \sigma_{y}} \exp \left[-\frac{\left(x-x_{s}\right)^{2}}{2 \sigma_{x}^{2}}-\frac{\left(y-y_{s}\right)^{2}}{2 \sigma_{y}^{2}}\right], \\
u_{s}(t) & =0.9 a_{1} \frac{1+\tanh \left(\frac{t-t_{o}}{\sigma_{t}}\right)}{2} \frac{1+\cos (\omega t)}{2}
\end{aligned}
$$

and $\left(x_{s}, y_{s}\right)$ denotes the center of injection ( $a_{1}$ being the speed of sound at the inlet). Thus, the forcing points were distributed using a compact and smooth function, and the injection was gradually activated so that spurious numerical disturbances can 


\begin{tabular}{c|c}
\hline Case & $\omega h_{1} / u_{1}$ \\
\hline $\mathbf{I}$ & 0.5 \\
$\mathbf{J}$ & 0.75 \\
$\mathbf{K}$ & 1.0 \\
$\mathbf{L}$ & 1.25 \\
$\mathbf{M}$ & 1.5 \\
$\mathbf{N}$ & 2.0 \\
$\mathbf{O}$ & 2.5 \\
\hline
\end{tabular}

Table 2: Frequency of mass flow injection in DNS. The base flow conditions are the same as Case $\mathbf{A}$; namely, $M_{1}=0.6, h_{2} / h_{1}=2.0$, and $R e=4000$ (refer to table 1).

be lessened. In actual computations, $x_{s}=-1.60$, $y_{s}=1.04, \sigma_{x}=0.08, \sigma_{y}=0.01, A_{o}=6.0 \times 10^{-3}$, $\sigma_{t}=0.2$, and $t_{o}=0.5$ were selected. Consequently, the momentum coefficient can be calculated as $C_{\mu} \equiv\left(\rho u_{s}^{2} h_{s}\right) /\left(\rho u_{1}^{2} h_{1}\right) \approx 0.52 \%, h_{s}$ being the corresponding slot width. The order of $C_{\mu}$ is comparable to experiments (e.g. Narayanan \& Banaszuk 2003). An attempt was made to minimize the forcing region and to locate it closer to the wall to simulate a slot on the wall.

Seven different forcing frequencies, $\omega$, were examined, and they are tabulated in table 2. The base flow conditions for these runs were the same as Case A shown in table 1.

\section{RESULTS AND DISCUSSION}

\section{VORTEX SHEDDING FREQUENCY FOR UNFORCED CASES}

Figure 8 displays vorticity contours computed from DNS at several instants in time for Case $\mathbf{A}$ (see table 1). The vorticity flux is accumulated somewhat downstream of the separation point, and a large-scale vortex is formed. While this vortex is pinched off and convected downstream, the next vortex is generated. The curved part of the wall is almost entirely contained within the separated vortex during this process.

Figure 9 depicts the time histories of stagnation pressure. It shows that the flow field has a characteristic time scale; in fact, our model, figure 5, captures such features. Referring to figure 8 , it is found that one period of vortex shedding roughly corresponds to an interval between troughs in the stagnation pressure profile: When the center of the vortex passes through the exit cross section, the stagnation pressure drops. Such a trend is consistent with our analysis.
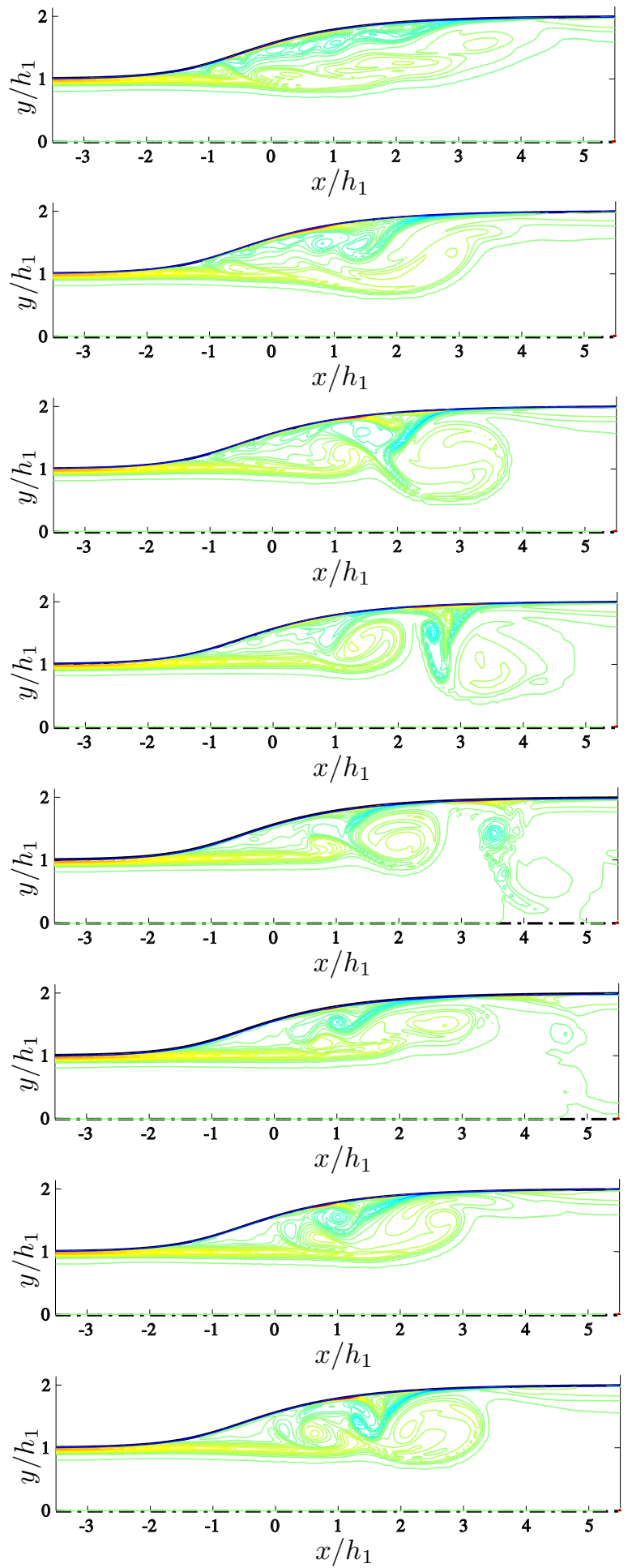

Figure 8: Vorticity evolution in a two-dimensional diffuser (unforced case, Case A). Vorticity contours computed from DNS are shown over time: $t u_{1} / h_{1}=$ 57.6, 60.0,62.4,64.8,67.2, 69.6, 72.0, and 74.4 from the top. Contour lines: $\omega_{\min }=-33.3, \omega_{\max }=33.3$, and the interval of $\Delta \omega=0.833$. 
To validate the inviscid model for the vortex shedding time period, a total of seven cases (Cases $\mathbf{A} \sim$ G) was simulated (refer to table 1) at various Mach numbers and area ratios. The time history of stagnation pressure at the exit was Fourier transformed in each case, and the local peak corresponding to the shedding time scale was chosen in the frequency domain. Figures 10 and 11 plot the ratios of the shedding time period from DNS to the predicted time scale given by (5) at different Mach numbers and area ratios, respectively. These figures show that the averaged shedding time periods are within a factor of two of those predicted by the model. The underestimates of the time scale by the model are likely caused by inaccurate estimates of the vortex density and size as well as the induced velocity $\alpha u_{1}$ to pinch off vortices. As discussed later, the absorption of circulation from the wall, $\lambda$, is relatively small $(\sim 10 \%)$ in the unforced cases.

\section{STAGNATION PRESSURE LOSS FOR UNFORCED CASES}

Next, the averaged stagnation pressure loss obtained from DNS is compared with the incompressible, inviscid $(\lambda=0)$ and fully-separated $\left(\overline{p_{w}}=p_{1}\right)$ model described before. The Mach number and area ratio dependence is shown in figures 12 and 13, respectively. Although the model captures the trend, the deviation is somewhat inconsistent. In DNS we attempted to optimize the computational cost by choosing the minimum Reynolds numbers to generate vortex shedding flow patterns. Therefore, the difference of the Reynolds numbers may affect flow patterns, such as the structure and the convective velocity of vortices. Note that the stagnation pressure loss associated with viscous dissipation is the order of $10^{-3}$ or less in all cases.

The depths of the stagnation pressure troughs in Case $\mathbf{A}$ are $4 \sim 10 \%$ (figure 9) and somewhat smaller than that of the Oseen vortex model $(\sim 11 \%)$ in figure 5 . On the other hand, the convective velocity of the vortices in DNS is nearly half $u_{2}$ (detailed values of the model coefficients will be given in table 3 and discussed later), while that estimated based on the model is $0.67 u_{2}$ (using (17) with $h_{2} / h_{1}=$ 2 and $\left.h_{3} / h_{1}=5 / 4\right)$. Hence, the troughs in DNS are wider than those predicted by the model. As a result, the unsteady part of the stagnation pressure loss is under-estimated by the model.

As mentioned in (22), the static pressure recovery provides a positive factor (i.e. $\overline{p_{w}}>p_{1}$ ) in the stagnation pressure recovery, which is ignored in the fully separated model. If we include this term, the model would under-estimate the stagnation pressure

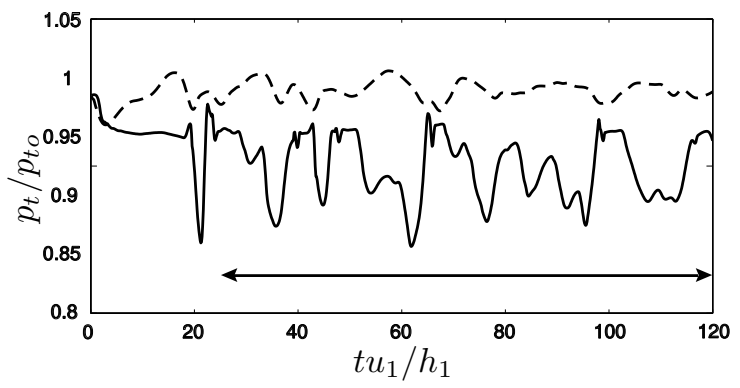

Figure 9: Time histories of stagnation pressure in Case A. Stagnation pressure was averaged over the cross section: $-\quad-\quad-$, inlet stagnation pressure $\left(x / h_{1}=-2.\right) ;-$, exit stagnation pressure $\left(x / h_{1}=3.\right) \cdot p_{t o} \equiv\left(1+\frac{\gamma-1}{2} M_{1}^{2}\right)^{\gamma /(\gamma-1)} p_{1}$.

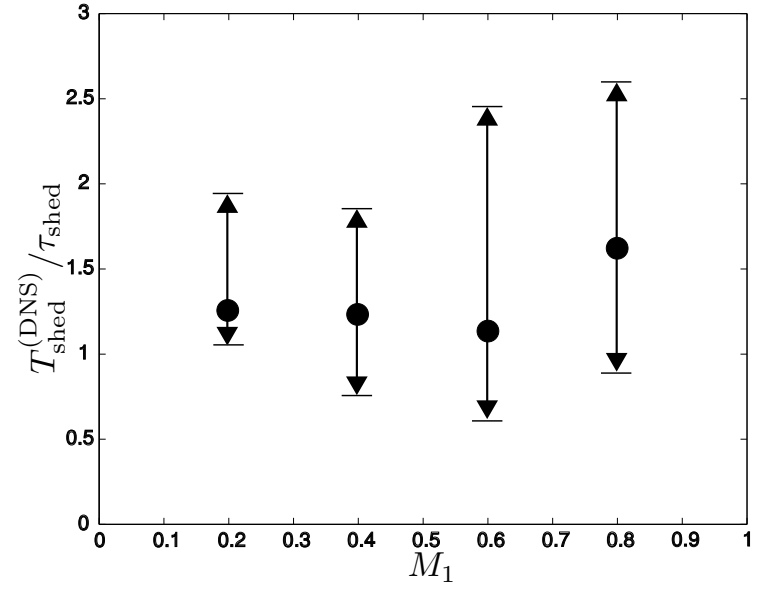

Figure 10: Mach number dependence of the vortex shedding period. Vortex shedding time periods from DNS are normalized by the shedding time scale given by (5). The results of Cases $\mathbf{B}, \mathbf{C}, \mathbf{A}$, and $\mathbf{D}$ are plotted from left to right. - denotes the peak time period, and the arrows denote the minimum and maximum time periods measured from intervals between troughs.

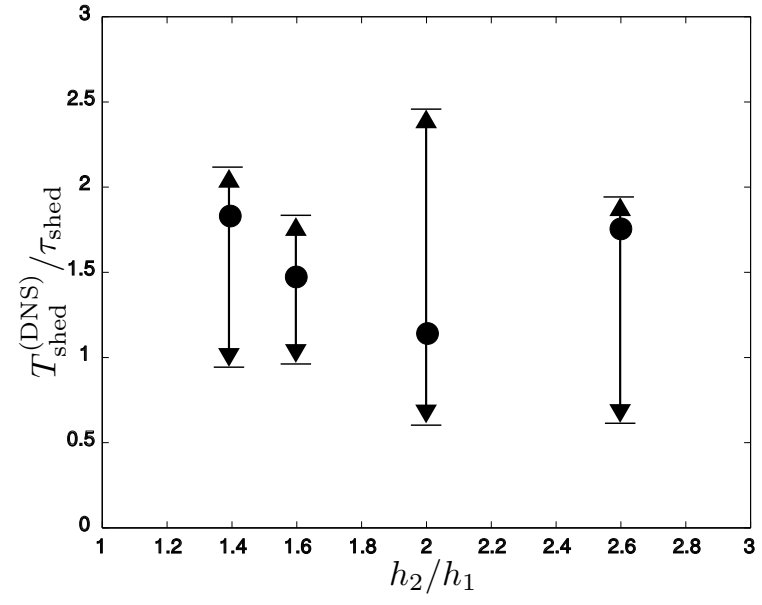

Figure 11: Area ratio dependence of the vortex shedding period. The results of Cases $\mathbf{E}, \mathbf{F}, \mathbf{A}$, and $\mathbf{G}$ are plotted from left to right. Notation is the same as figure 10 . 
loss in most cases. In fact, this factor is partially canceled by the under-estimate of the unsteady part mentioned above.

Referring to figures 10 and 11, the stagnation pressure loss tends to exceed the prediction in figures 12 and 13 as the fluctuation of the shedding time period becomes wider (refer to the distances between the arrows in figures 10 and 11). When the accumulated circulation is clearly washed out due to the pinchingoff process, the unsteady pattern approaches stationary and stagnation pressure is better restored.

To assess the viscous effect on the wall, we calculated the vorticity flux on the upper wall $\left(x / h_{1} \in\right.$ $[-2,3])$ for Case $\mathbf{A}$ and exhibit in figure 14. Referring to figure 8 , it is clear that vorticity is mainly absorbed near the leading edge of the vortex. When the vortex convects downstream, the pressure on the wall $\left(p_{2}\right)_{\text {wall }}$ decreases; hence, vorticity is produced at the wall as derived from (22). The net rate of absorption in the time interval denoted in figure 9 is about $11 \%$; thus, the viscous effect is relatively small in the unforced case.

\section{PERIODIC MASS INJECTION}

The series of figure 15 shows the evolution of the vorticity contour for a forced case (Case $\mathbf{L}$, which corresponds to the optimum forcing frequency as shown later). Compared with the unforced case (figure 8), the size of the vortices is smaller and their convective velocity is higher in this case. Near the separation point, the next vortex is continuously generated and pinched off before it is fully developed. Thus, the periodic mass injection tends to reduce $\alpha$ and $\kappa$ in (19) and suppresses the unsteady part of stagnation pressure loss.

Figures 16 and 17 compare the averaged static pressure field for the unforced (Case A) and forced (Case $\mathbf{L})$ cases, respectively. They demonstrate that the reattachment point shifts upstream in the forced case although the apparent separation point does not move in figure 15. This helps static pressure recovery on the wall and lessens the steady part of stagnation pressure loss.

Figure 18 depicts the time histories of stagnation pressure at the inlet and exit for Case $\mathbf{L}$. Compared to the unforced case (figure 9), these profiles clearly become periodic at the forcing frequency (i.e. frequency locking). Moreover, the forced case shows sharper stagnation pressure drops and a higher offset; namely, the convective velocity is increased and static pressure on the wall is restored.

Figure 19 compares the stagnation pressure recovery between the unforced and forced cases over a range of frequencies. Periodic mass injection pro-

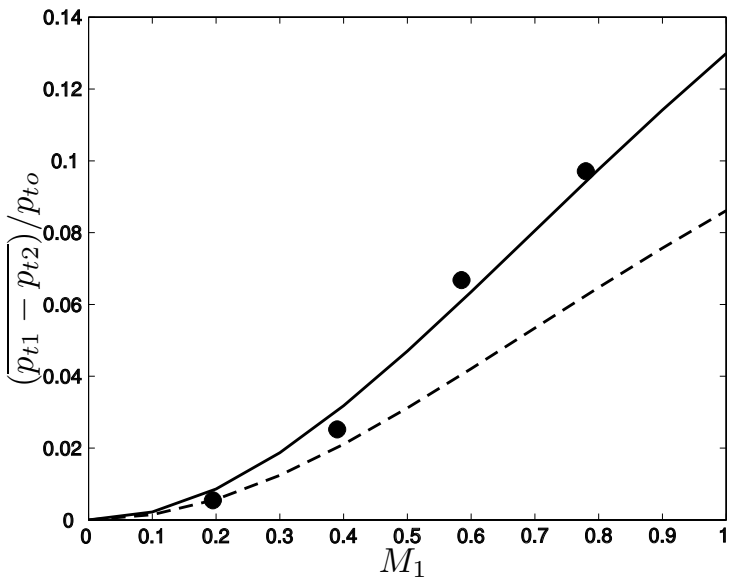

Figure 12: Mach number dependence of the stagnation pressure loss. Time averaged stagnation pressure loss calculated from DNS is compared with the fully separated model in the incompressible limit: - total stagnation pressure loss given by (19) $\left(\alpha=1, \beta=1, \lambda=0, \overline{p_{w}}=p_{1}\right.$, and $\kappa=0.33$ are assumed); - - - , steady part given by (13); $\bullet$, DNS results (Cases $\mathbf{B}, \mathbf{C}, \mathbf{A}$, and $\mathbf{D}$ from left to right). The actual averaged inflow Mach numbers are plotted for DNS.

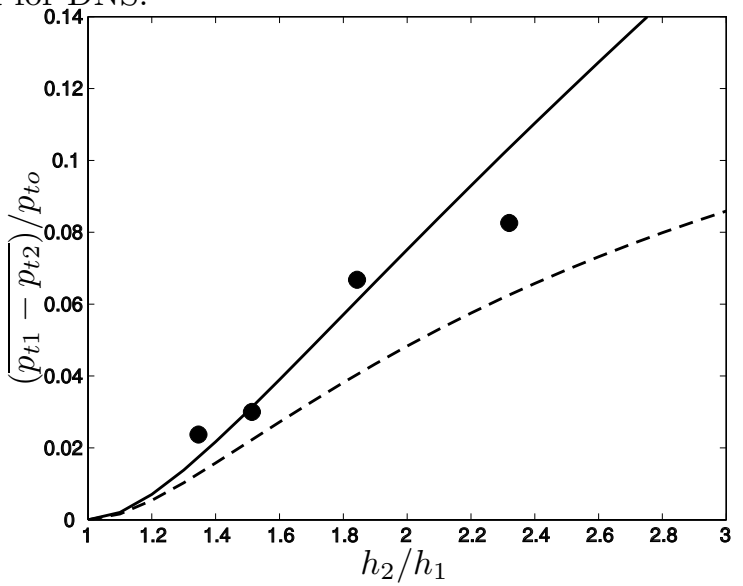

Figure 13: Area ratio dependence of the stagnation pressure loss. Notation is the same as figure 12. DNS results, Cases $\mathbf{E}, \mathbf{F}, \mathbf{A}$, and $\mathbf{G}$, are plotted from left to right. The actual area ratio between the inlet and the exit stations indicated in table 1 is used. $h_{3}=\frac{1}{2}\left(\frac{h_{1}+h_{2}}{2}+h_{2}\right)$ is assumed to evaluate $\kappa$.

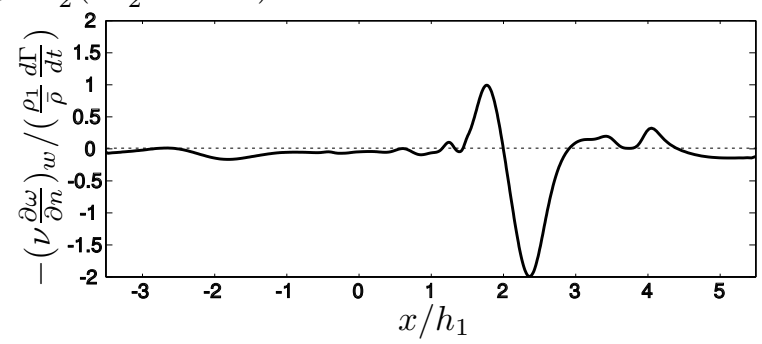

Figure 14: Instantaneous vorticity flux from the wall in Case A. Vorticity flux from the upper wall is calculated based on (21) at $t u_{1} / h_{1}=67.2$ in Case $\mathbf{A}$ (see figure 8). Production is taken to be positive, and absorption to be negative. 

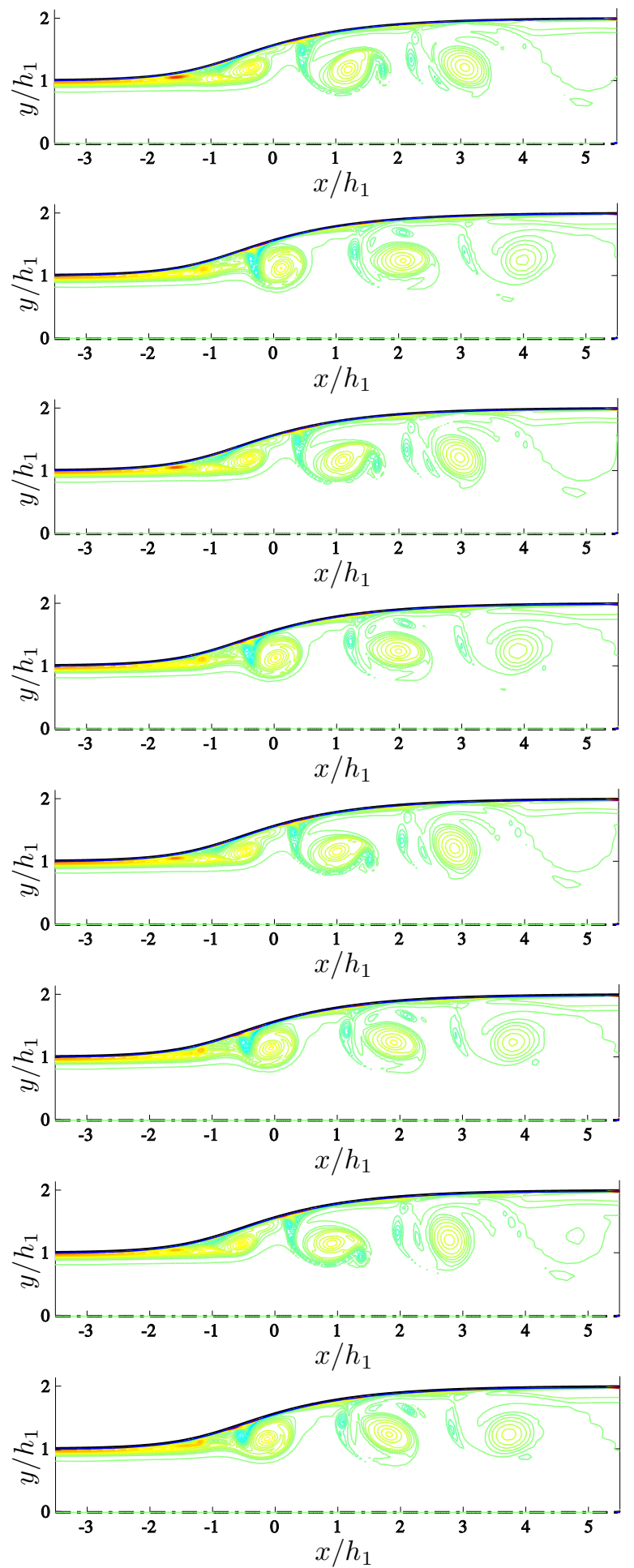

Figure 15: Vorticity evolution in a two-dimensional diffuser

at the optimum forcing frequency $\left(\omega h_{1} / u_{1}=1.25\right.$, Case $\mathbf{L})$. Notation is the same as figure 8: $t u_{1} / h_{1}=$ $57.6,60.0,62.4,64.8,67.2,69.6,72.0$, and 74.4 from the top. vides better stagnation pressure recovery to an appreciable degree $(2 \sim 4 \%)$ in all cases. (Note that the direct increase of stagnation pressure due to mass injection is approximately $\rho \overline{u_{s}^{2}} h_{s} /\left(2 p_{t o} h_{1}\right) \sim$ $\left.2 \times 10^{-3}\right)$. In particular, frequency locking, which is observed at nearly the natural shedding frequency or somewhat higher, results in the most substantial improvement in stagnation pressure recovery. At even higher frequencies, vortex pairing is observed, and the stagnation pressure recovery is deteriorated. An example of vortex pairing is displayed in figure 20 .

The optimum frequency is slightly less than twice the natural shedding frequency. In fact, the order of this frequency agrees with the non-dimensional frequency of $F^{+} \equiv f L / u_{\infty} \sim 1$ ( $L$ is a characteristic length and $u_{\infty}$ the free-stream velocity, which we take here as $\left(h_{2}-h_{1}\right)$ and $u_{1}$, respectively). This frequency scale is used in various applications (Seifert, Darabi, \& Wygnanski 1996). Interestingly, even if the frequency locking occurs, the higher frequency does not necessarily lead to better performance.

These results also agree with the finding that periodic forcing is most effective when the vortex is reduced in size by a factor of one-third to unity as compared with the natural vortex size (Seifert, Darabi, \& Wygnanski 1996; Seifert \& Pack 1999). However, counter to the interpretation given in previous studies, the forcing mechanism is not directly related to instabilities of the shear layer near the separation point. As shown in figure 19, the most unstable frequency of the shear layer estimated from the linear stability analysis (the mean velocity profile is taken from $x / h_{1}=-0.5$ in Case $\mathbf{A}$ ) is found to be several times higher than the optimum frequency. In experiments, the most unstable frequency should be even higher as the boundary layer becomes thinner. Our theoretical discussion also implies that the local shear layer instability does not explicitly govern the forcing mechanism.

Table 3 shows various parameters associated with the model computed from DNS. The correlation between $\overline{p_{t 2}} / \overline{p_{t 1}}$ and $\lambda$ demonstrates that the absorption of circulation helps improve stagnation pressure recovery. Even if no frequency locking occurs (Case I), the rate of absorption in the forced case is substantially higher than that in the unforced case; similarly, the stagnation pressure recovery is also higher in the forced cases. Recall that the mass injection in this study has always a non-negative momentum flux. In the optimum case (Case $\mathbf{L}$ ), the rate of absorption is as much as $60 \%$. As seen in figures 14 , a small vortex generated near the separation point locally creates an adverse pressure gradient (see (21)) and helps absorb vorticity from the wall. 


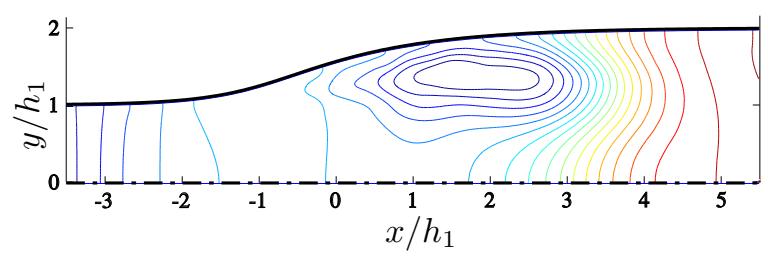

Figure 16: Time averaged pressure contour for the unforced case (Case A). Contour level: $\bar{p}_{\min } / p_{1}=$ $1.00, \bar{p}_{\max } / p_{1}=1.16$ with the interval of $\Delta \bar{p} / p_{1}=$ 0.01 .

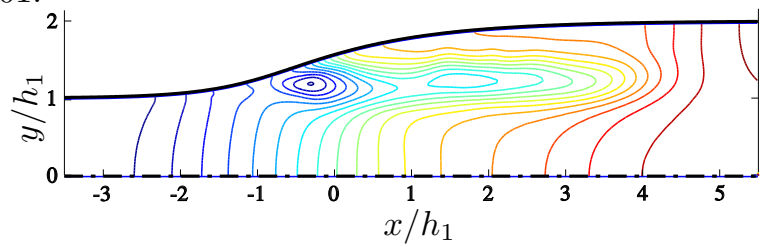

Figure 17: Time averaged pressure contour for the forced case (Case $\mathbf{L}$ ). Notation is the same as figure 16.

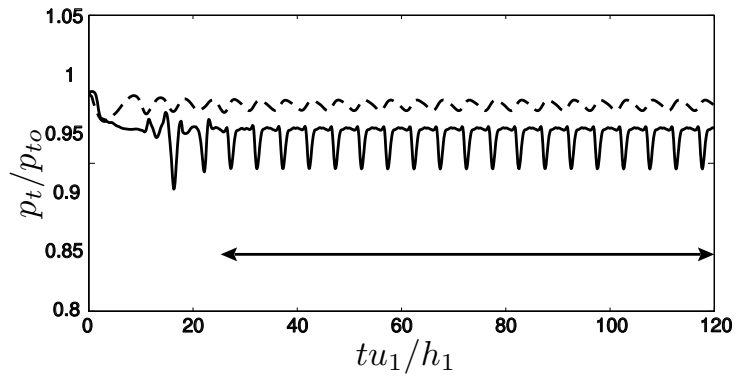

Figure 18: Time histories of stagnation pressure at the optimum forcing frequency $\left(\omega h_{1} / u_{1}=1.25\right.$, Case $\mathbf{L})$. Notation is the same as figure 9 .

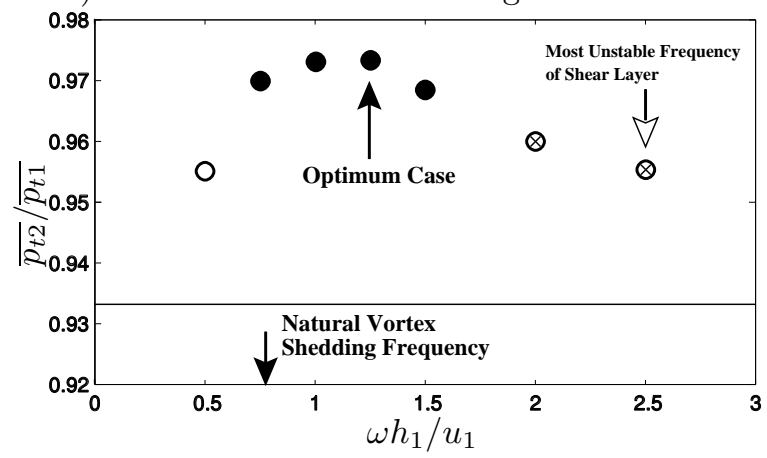

Figure 19: Comparison of the stagnation pressure recovery at different forcing frequencies. The ratios of the averaged stagnation pressures at the exit $\left(x / h_{1}=3\right.$. $)$ to the inlet $\left(x / h_{1}=-2\right.$. $)$ are plotted (Cases $\mathbf{I} \sim \mathbf{O}$ from left to right). The horizontal line denotes the unforced case (Case $\mathbf{A})$. $\bullet$ indicates that frequency locking occurs, and $\otimes$ indicates that vortex pairing occurs although the vortices are pinched off at the forcing frequency.

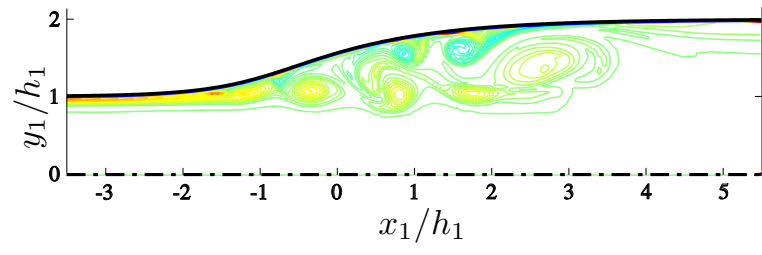

Figure 20: A snapshot of vortex pairing. Vorticity contours are drawn (Case $\mathbf{O}$ ). Notation is the same as figure 8.

\begin{tabular}{c|cccc}
\hline Case & $\omega h_{1} / u_{1}$ & $\overline{p_{t 2}} / \overline{p_{t 1}}$ & $\overline{p_{w}} / p_{1}$ & $\lambda$ \\
\hline $\mathbf{A}$ & unforced & 0.933 & 1.049 & 0.113 \\
$\mathbf{I}$ & 0.5 & 0.955 & 1.055 & 0.402 \\
$\mathbf{J}$ & 0.75 & 0.970 & 1.065 & 0.588 \\
$\mathbf{K}$ & 1.00 & 0.973 & 1.070 & 0.603 \\
$\mathbf{L}$ & 1.25 & 0.973 & 1.074 & 0.604 \\
$\mathbf{M}$ & 1.50 & 0.968 & 1.075 & 0.536 \\
$\mathbf{N}$ & 2.00 & 0.960 & 1.071 & 0.439 \\
$\mathbf{O}$ & 2.50 & 0.955 & 1.065 & 0.388 \\
\hline \hline Case & $\alpha$ & $\beta$ & $1-\alpha \kappa$ & $h_{3} / h_{1}$ \\
\hline $\mathbf{A}$ & 1.154 & 0.837 & 0.531 & 1.325 \\
$\mathbf{I}$ & 0.920 & 0.886 & 0.578 & 1.180 \\
$\mathbf{J}$ & 0.690 & 1.045 & 0.678 & 1.097 \\
$\mathbf{K}$ & 0.547 & 0.986 & 0.771 & 1.163 \\
$\mathbf{L}$ & 0.452 & 0.936 & 0.782 & 1.237 \\
$\mathbf{M}$ & 0.378 & 1.025 & 0.746 & 1.271 \\
$\mathbf{N}$ & 0.519 & 0.920 & 0.663 & 1.301 \\
$\mathbf{O}$ & 0.664 & 1.000 & 0.557 & 1.252 \\
\hline
\end{tabular}

Table 3: Frequency dependence of each parameter measured from DNS. $\overline{p_{w}}$ and $\lambda$ were directly computed on the upper wall (using (21) for $\lambda$ ). $\alpha, \beta$, and $(1-\alpha \kappa)$ were calculated from three samples of vortices passing through the exit cross section $\left(x_{1} / h_{1}=3\right.$. $)$ for each case. A single vortex was defined as a simply supported region of the local vorticity up to $2 \%$ of the peak vorticity.

Attention should be paid to the rate of absorption, $\lambda$, and the convective velocity, $(1-\alpha \kappa)$, which are lower in Case $\mathbf{M}$ than Case $\mathbf{L}$. When a vortex is pinched off due to forcing, its center stays closer to the wall for a smaller vortex. In fact, $h_{3} / h_{1}$ measured in DNS demonstrates this trend. Hence, the convective velocity is reduced from (17), and the pressure deficit on the wall is enhanced, i.e. the rate of absorption is decreased from (22). This effect seems to provide an upper limit on the diminution of stagnation pressure loss with decreasing $\alpha$. In the unforced case, vortices tend to be pinched off further downstream, and, in turn, the vortex center is again close to the wall regardless of the size of the vortex.

Finally, figure 21 depicts the estimated stagnation 


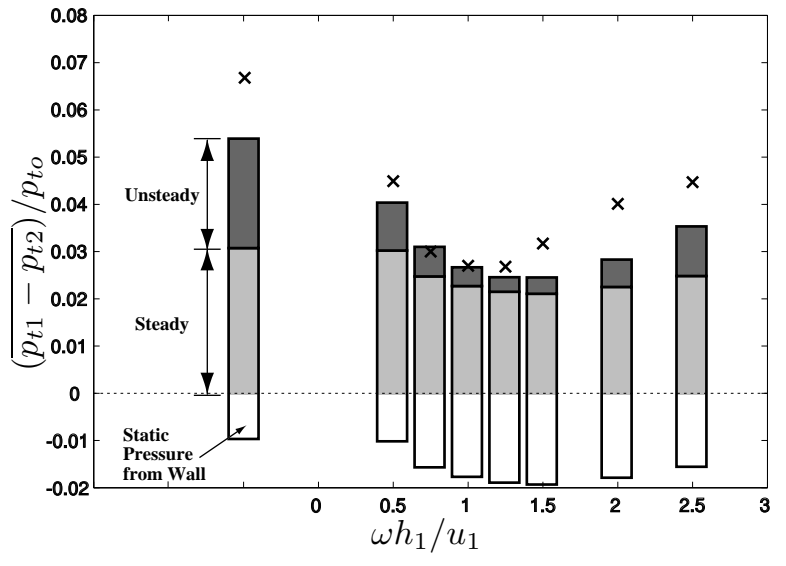

Figure 21: Estimated stagnation pressure loss from each component. Each component of the stagnation pressure loss is displayed in a bar chart. Parameters in table 3 are substituted into (19). The actual total stagnation pressure directly computed from DNS is denoted by $\times$. The unforced case (Case $\mathbf{A}$ ) is also plotted on the left.

pressure loss from each component in the model. It indicates that the unsteady part of the stagnation pressure loss is substantially reduced near the optimum frequency. As the forcing frequency is further increased (at $\omega h_{1} / u_{1}=2.0$ in Case $\mathbf{N}$ ), $\alpha$ is nearly doubled due to vortex pairing. Likewise, at $\omega h_{1} / u_{1}=2.5$ in Case $\mathbf{O}, \alpha$ is tripled or more due to multiple vortex pairings. Static pressure $\overline{p_{w}} / p_{1}$ is restored for smaller circulation of vortices, but this contribution is weaker than the unsteady part.

The under-estimates of the net stagnation pressure loss are presumably caused by the compressible effects as well as the inaccuracy of measurement for $\alpha$ and $(1-\alpha \kappa)$, particularly when vortex parings occur (Cases $\mathbf{N}$ and $\mathbf{O}$ ) or the flow pattern becomes fully unsteady (Cases A and $\mathbf{I}$ ). In addition, counterrotating vortices (small ones can be observed in figures 8 and 15), which are ignored in this chart, can deteriorate the stagnation pressure recovery as well.

\section{CONCLUSIONS}

A reduced order model which captures the vortex shedding phenomenon and estimates stagnation pressure loss in a diffuser is developed in twodimensions. DNS is performed at various Mach numbers and area ratios to validate the model. The characteristic frequency of vortex shedding is successfully scaled by accounting for the net vorticity accumulation. Stagnation pressure loss is also estimated fairly well based on an incompressible flow although the unsteady part of the loss tends to be under-estimated compared with the DNS results.
To suppress the stagnation pressure loss, we introduce periodic mass injection to simulate a synthetic jet. Frequency locking occurs at nearly the natural vortex shedding frequency or somewhat higher where the most substantial improvement is achieved in stagnation pressure recovery $(3 \sim 4 \%)$. Periodic injection continuously generates vortices downstream of the separation point. These vortices locally create an adverse pressure gradient and enhance the absorption of circulation from the wall.

The analyses based on the incompressible model together with the DNS results imply that the key to improve the stagnation pressure recovery is the following: (1) absorb more circulation from the wall (increase $\lambda$ ); $(2)$ reduce circulation of one vortex (decrease $\alpha) ;(3)$ increase the convective velocity of vortices (decrease $\kappa)$; (4) increase the static pressure recovery on the wall (increase $\overline{p_{w}}$ ). These parameters are not independent; in fact, three parameters, $\lambda, \alpha$, and $\overline{p_{w}}$, tend to simultaneously shift to the preferable direction by increasing the forcing frequency until frequency locking breaks. Although the current model can give a crude estimate of $\alpha$ (and maybe $\kappa)$, it cannot predict $\lambda$ and $\overline{p_{w}}$. To actually estimate these parameters for forced cases, we need to analyze vortex dynamics in each specific flow geometry. Nonetheless, the DNS results show that the optimum frequency occurs at somewhat higher than the natural vortex shedding frequency, which is qualitatively consistent with the experimental facts.

It should be emphasized that this study focuses on the cases in which the separation point barely moves due to the forcing. For some applications the adverse pressure gradient is relatively gentle so that the mass injection can delay the separation point and provide better static pressure recovery. In fact, classical active flow control techniques, such as steady tangential blowing or boundary layer suction, try to attach the boundary layer. In those studies, the slope angle of the diffuser $\theta$ is considered to be an important parameter. However, the model developed in this study implies that the area ratio governs the largescale flow unsteadiness for rapidly expanding diffusers. Furthermore, this study demonstrates that by pinching off vortices more frequently, we can suppress a substantial part of the unsteady stagnation pressure loss. As a result, the stagnation pressure recovery is much improved even though the boundary layer remains separated.

Although we have only simulated laminar diffuser flows, the boundary layer becomes turbulent under practical conditions. However, the model developed in this study is independent of the Reynolds number and boundary layer thickness, except that the rate 
of absorption can change. This explains the experimental results that the peak frequency varies weakly with Reynolds numbers (Seifert \& Pack 1999). We recognize that the model and DNS developed here lack several features of real diffuser flows. The most obvious limitation is the two-dimensionality although the results, particularly the forcing strategies, should still be commonly applicable to threedimensional diffusers with spanwise coherent vortex shedding (c.f. Kaltenbach, et al. 1999).

\section{ACKNOWLEDGMENTS}

The DNS code was developed in collaboration with Dr. S. Pirozzoli and Mr. J. Fung. The authors also would like to thank Drs. D. MacMartin, J. Paduano, T. P. Hynes, and Profs. A. P. Dowling, R. Murray, A. Leonard, and H. Nagib for fruitful discussions. This research was sponsored by the DARPA (Defense Advanced Research Project Agency) program (the contract number F49620-00-C-0035).

\section{REFERENCES}

Back, L. \& Cuffel, R. 1982 Experimental investigation of turbulent wall-jets in the presence of adverse pressure gradients in a rectangular diffuser. Int. J. Heat Mass Transfer, 25, 871-887.

Freund, J. B. 1997 Proposed inflow/outflow boundary condition for direct computation of aerodynamic sound. AIAA J. 35, no.4, 740-742.

Gharib, M., Rambod, E., \& Shariff, K. 1998 A universal time scale for vortex ring formation. J. Fluid Mech. 360, 121-140.

Glezer, A. \& Amitay, M. 2002 Synthetic jets. Annu. Rev. Fluid Mech. 34, 503-529.

Hamstra, J. W., Miller, D. N., Truax, P. P., Anderson, B. A., \& Wendt, B. J. 2000 Active inlet flow control technology demonstration. in International Congress of the Aeronautical Sciences. ICAS-20006.11.2.

Kaltenbach, H.-J., Fatica, M., Mittal, R., Lund, T. S., \& Moin, P. 1999 Study of flow in a planar asymmetric diffuser using large-eddy simulation. J. Fluid Mech. 390, 151-185.

Koumoutsakos, P., Leonard, A., \& Ṕepin, F. 1994 Boundary conditions for viscous vortex methods. J. Comput. Phys. 113, 52-61.

Lele, S. K. 1992 Compact finite difference schemes with spectral-like resolution. J. Comput. Phys., 103, no.1, 16-42.
MacMartin, D. G., Murray, R. M., Verma, A., \& Paduano, J. D. 2001 Active control of integrated inlet/compression systems: Initial results. ASME Fluid Engng. FEDSM 2001-18275.

Narayanan, S. \& Banaszuk, A. 2003 Experimental study of a novel active separation control approach. AIAA paper, AIAA 2003-0060.

Nicoll, W. B. \& Ramaprian, B. R. 1970 Performance of conical diffusers with annular injection at inlet. Trans. ASME: J. Basic Engng. 92, no.4, 827-835.

Nishi, M., Yoshida, K., \& Morimitsu, K. 1998 Control of separation in a conical diffuser by vortex generator jets. JSME: Int. J. Series B, 41, no.1, 233238 .

Oseen, C. W. 1912 Über Wirbelbewegung in einer reibenden Flüssigkeit. Ark. f. Mat. Astron. Fys. 7,14 .

Pierrehumbert, R. T. 1980 A family of steady, translating vortex pairs with distributed vorticity. J. Fluid Mech. 99, pt.1, 129-144.

Poinsot, T. J. \& Lele, S. K. 1992 Boundary conditions for direct simulations of compressible viscous flows. J. Comput. Phys. 101, no.1, 104-129.

Rao, D. M. 1971 A method of flow stabilisation with high pressure recovery in short, conical diffusers. J. Roy. Aeronaut. Soc. Technical notes, 75, 336-339.

Reneau, L. R., Johnston, J. P., \& Kline, S. J. 1967 Performance and design of straight, two-dimensional diffusers. Trans. ASME: J. Basic Engng. 89, no.4, 141-150.

Saffman, P. G. 1992 Vortex Dynamics, Cambridge University Press.

Salmon, J. T., Bogar, T. J., \& Sajben, M. 1983 Laser Doppler velocimeter measurements in unsteady, separated, transonic diffuser flows. AIAA J. 21, no.12, 1690-1697.

Seifert, A., Darabi, A., \& Wygnanski, I. 1996 Delay of airfoil stall by periodic excitation. J. Aircraft, 33, no.4, 691-698.

Seifert, A. \& Pack, L. G. 1999 Oscillatory control of separation at high Reynolds Numbers. AIAA J. 37, no.9, 1062-1071.

Schlichting, H. 1960 Boundary Layer Theory, 4th ed. (translated by J. Kestin), McGraw-Hill.

Welsh, M. C. 1976 Flow control in wide-angled conical diffusers. Trans. ASME: J. Fluids Engng. 98, no.4, 728-735. 